\title{
On completeness of the Bergman metric and its subordinate metric
}

\author{
(Carathéodory differential metric/Weil-Petersson metric/Teichmüller space)
}

\section{KYONG T. HAHN}

Department of Mathematics, The Pennsylvania State University, University Park, Pa. 16802

ABSTRACT It is proved that on any bounded domain in the complex Euclidean space $C^{n}$ the Bergman metric is always greater than or equal to the Carathéodory distance. This leads to a number of interesting consequences. Here two such consequences are given. (i) The Bergman metric is complete whenever the Carathéodory distance is complete on a bounded domain. (ii) The Weil-Petersson metric is not uniformly equivalent to the Bergman metric in the Teichmüller space $T(g)$ of any Riemann surface of genus $\boldsymbol{g} \geq 2$.

Let $D$ be any bounded domain in the complex Euclidean space $C^{n}(n \geq 1)$. Then $D$ admits both the Bergman metric $s_{D}$ and the Caratheodory differential metric $\alpha_{D}$. The main purpose of this note is to prove the following

THEOREM 1. Let $D$ be a bounded domain in $C^{n}$. For each $z \in D$ and each $\xi \in C^{n}$

$$
\alpha_{D}(z \xi) \leq s_{D}(z \xi) \text {. }
$$

Let $\rho_{D}$ and $d_{D}$ be the distance functions on $D$ which are induced from $\alpha_{D}$ and $s_{D}$, respectively. Then the Carathéodory distance $c_{D}$ satisfies:

$$
c_{D} \leq \rho_{D} \leq d_{D}
$$

From this observation, we have

THEOREM 2. Let $D$ be any bounded domain $C^{n}$. Then the Bergman metric $s_{D}$ is complete in $D$ if the Caratheodory distance $c_{D}$ is complete.

Recently, S. Wolpert in ref. 1 and T. Chu have independently proved that the Weil-Petersson metric is not complete in the Teichmüller space $T(g)$ of a compact Riemann surface of genus $g \geq 2$. On the other hand, it follows from Theorem 2 and the completeness of the Carathéodory distance in $T(g)$ (see ref. 2 ) that the Bergman metric is complete in $T(g)$. Therefore, we conclude the following.

THEOREM 3. In the Teichmïller space $T(g)$ of any Riemann surface of genus $g \geq 2$, the Weil-Petersson metric is not uniformly equivalent to the Bergman metric.

For the proof of Theorem 1 we need the following

LEMMA. Let $f$ be a holomorphic function on $D$ satisfying $|f(z)| \leq M, M>0$. Then for each $z \in D$ and each $\xi \in C^{n}$,

$$
\left|\sum_{\nu=1}^{n} \frac{\partial f}{\partial z_{\nu}} \xi_{\nu}\right|^{2} \leq M^{2} \sum_{\alpha, \beta=1}^{n} \frac{\partial^{2} \log K_{D}(z \bar{z})}{\partial z_{\alpha} \partial \bar{z}_{\beta}} \xi_{\alpha} \xi_{\beta,}
$$

where $K_{D}$ is the Bergman kernel function of $D$.

PROOF: Set $a(t)=f(t) K_{z}(t), \quad b(t)=\Sigma_{\nu=1}{ }^{n}$ $\xi_{\nu}\left(\partial / \partial \bar{z}_{\nu}\right)\left[K_{z}(t) / K_{z}(z)\right]$, and $(a, b)_{D}=\int_{D} a \bar{b} d \omega$, where $d \omega$ is the Lebesgue measure in $D$ and $K_{z}(t)=K_{D}(t, \bar{z})$.
By the Schwarz inequality

$$
\left|(a, b)_{D}\right|^{2} \leq(a, a)_{D}(b, b)_{D} .
$$

Using the reproducing property of $K_{D}$ (ref. 3), we have

$$
(a, a)_{D}=\left(f K_{z}, f K_{z}\right)=\left(f \bar{f} K_{z}, K_{z}\right) \leq M^{2} K_{D}(z \bar{z}),
$$

$$
\begin{aligned}
(b, b)_{D}= & \sum_{\mu, \nu=1}^{n} \xi_{\mu} \xi_{\nu}\left(\frac{\partial}{\partial \bar{z}^{\nu}}\left(\frac{K_{z}}{K_{z}(z)}\right), \frac{\partial}{\partial \bar{z}^{\mu}}\left(\frac{K_{z}}{K_{z}(z)}\right)\right) \\
=\sum \xi_{\mu} \xi_{\nu} K_{z}^{-3}(z) & {\left[K_{z}(z) \frac{\partial^{2} K_{z}(z)}{\partial z_{\mu} \partial \bar{z}_{\nu}}-\frac{\partial K_{z}(z)}{\partial z_{\mu}} \frac{\partial K_{z}(z)}{\partial \bar{z}_{\nu}}\right] } \\
& =\frac{1}{K_{D}(z, \bar{z})} \sum_{\mu, \nu=1}^{n} \frac{\partial^{2} \log K_{D}(z, \bar{z})}{\partial z_{\mu} \partial \bar{z}_{\nu}} \xi_{\mu} \xi_{\nu}
\end{aligned}
$$

and

$$
\begin{aligned}
(a, b)_{D}=\sum_{\nu=1}^{n} \xi_{\nu}\left(f K_{z}, \frac{\partial}{\partial \bar{z}_{\nu}} \frac{K_{z}}{K_{z}(z)}\right) \\
=\sum_{\nu=1}^{n} \xi_{\nu} \frac{\partial}{\partial z_{\nu}}\left(\frac{f(z) K_{D}(z, \bar{z})}{K_{D}(z, \bar{z})}\right)=\sum_{\nu=1}^{n} \xi_{\nu} \frac{\partial f}{\partial z_{\nu}} .
\end{aligned}
$$

From [1] together with [2], [3], and [4], Lemma follows.

Theorem 1 is now an immediate consequence of Lemma when we recall that the Caratheodory differential metric of $D$ is given (see ref. 4) by

$$
\alpha_{D}(z, \xi)=\sup \left\{\left|\sum_{\nu=1}^{n} \frac{\partial f}{\partial z_{\nu}} \xi_{\nu}\right|: f \in H(D, \Delta)\right\},
$$

where $H(D, \Delta)$ denotes the class of holomorphic functions $f$ on $D$ with values in the unit disc $\Delta$ in $C$.

It should be noted that the method of the proof of the above lemma was essentially due to K. H. Look in ref. 5 .

The author acknowledges that the above simplification of the proof of Theorem 1 was first suggested by J. Burbea.

1. Wolpert, S. (1975) "Non-completeness of the Weil-Petersson metric for Teichmüller space," Pac. J. Math. 61, 573-577.

2. Earle, C. J. (1974) "On the Caratheodory metric in Teichmüller spaces," Ann. of Math. Studies, No. 79 (Princeton University Press, Princeton, N.J.), pp. 99-103.

3. Bergman, S. (1970) "The kernel function and conformal mapping," Math. Surveys 5 (American Mathematical Society, Providence, R.I.), 2nd ed.

4. Reiffen, H. (1965) "Die Carathéodory Distanz und ihre zugehörige differential Metrik," Math. Ann. 161, 315-324.

5. Look, K. H. (1958) "Schwarz lemma and analytic invariants," Scientia Sinica 7, 453-504. 